\title{
Caraterização de Alunos Identificados em Risco na Leitura num Sistema de Triagem Universal
}

\author{
- Paula Marisa Fortunato Vaz* \\ - Ana Paula Loução Martins *
}

\section{Resumo}

Esta pesquisa pretendeu caraterizar alunos em risco na leitura, nomeadamente o seu nível de compreensão e os fatores de risco intrínsecos, parentais e contextuais que lhes estão associados. Participaram 82 alunos portugueses do 3ㅇa ano de escolaridade. Coletaram-se dados com uma prova de monitorização com base no currículo e uma grelha de identificação de fatores de risco. Concluiu-se que, na fase inicial do ano letivo, os resultados médios do nível de compreensão da leitura dos alunos que nunca mostraram risco é superior ao triplo dos resultados médios daqueles que ficaram em risco durante todo o ano letivo, e no final do ano letivo é sensivelmente duas vezes mais elevado; os alunos que nunca evidenciaram risco crescem mais semanalmente do que os alunos que evidenciaram risco em todo o ano letivo; os problemas na articulação, o atraso no desenvolvimento da linguagem e a ausência de experiências de leitura a pares com os progenitores, destacam-se como fatores de risco.

Palavras-chave: Triagem universal, leitura, fatores de risco, monitorização com base no currículo.

Doutora em Estudos da Criança, especialidade de Educação Especial, Universidade do Minho, Braga. Professora Adjunta, Centro de Investigação em Educação Básica, Instituto Politécnico de Bragança, Portugal; E-mail: paulavaz@ipb.pt.

** Doutora em Estudos da Criança, especialidade de Educação Especial, Universidade do Minho, Braga. Professora Auxiliar, Centro de Investigação em Educação, Instituto de Educação, Universidade do Minho, Braga, Portugal. Email: apmartins@ie.uminho.pt. 


\section{Introdução}

As escolas devem implementar um modelo de prevenção que assegure a existência de um ensino de qualidade para todos os alunos (prevenção primária), intervenções cientificamente elaboradas para os alunos que não respondem à prevenção primária (prevenção secundária) e o fornecimento de serviços individualizados intensivos, para aqueles que não responderam nem a um ensino de qualidade, nem às subsequentes intervenções (prevenção terciária) (BRADLEY; DANIELSON; HALLAHAN, 2002). De fato, a investigação tem mostrado ser adequada a existência de um apoio pró-ativo baseado na implementação de um sistema integrado de deteção precoce e progressivos níveis de apoio. O objetivo é que as escolas de uma forma organizada, eficiente e efetiva lidem com muitas das necessidades e características dos alunos e do contexto social onde se inserem. Segundo a opinião dos investigadores, este modelo, que é designado de Resposta à Intervenção, apresenta várias vantagens gerais (MARTINS, 2006). Aquela que aqui se sublinha relaciona-se com a possibilidade de se identificarem e apoiarem precocemente (em anos iniciais da escolaridade, bem como no início do ano letivo) os alunos em risco (FUCHS et al., 2002a; FUCHS 2002b; FUCHS 2003; SCRUGGS, 2003; VAUGHN, 2002). A existência de um sistema de triagem universal permite monitorizar o progresso dos alunos, a eficácia do ensino prestado no contexto da turma e/ou do apoio (FUCHS, 2002). Paralelamente é pertinente que durante este processo se aprofunde o conhecimento acerca dos alunos em risco na leitura, nomeadamente no que respeita aos fatores de risco que lhes podem estar associados, conhecimento este que, aliado à informação obtida com a monitorização vai ser útil na identificação precoce das suas dificuldades, na preparação de uma intervenção adequada (MCCARDLE; SCARBOROUGH; CATTS, 2001; SCARBOROUGH, 1998) e, portanto, na prevenção de dificuldades mais acentuadas (BURNS; GRIFFIN; SNOW, 1999).

\section{O processo de triagem universal do modelo de resposta à intervenção: A monitorização da leitura e os fatores de risco na leitura}

O Modelo de Resposta à Intervenção caracteriza-se por ser um sistema de prevenção, de ensino e de intervenções de apoio desenvolvido em três ou quatro níveis de intensidade, sendo o primeiro aquele que é mais utilizado pelas instituições de ensino 
(FLETCHER; VAUGHN, 2009; FUCHS; FUCHS, 2008; JOHNSON et al., 2006; CENTER RESPONSE TO INTERVENTION, 2012; NJCLD, 2005; VAUGHN; BOS, 2009). Na Figura 1 apresentam-se as características de um modelo com três níveis de prevenção.

Figura 1 - Modelo de Resposta à Intervenção

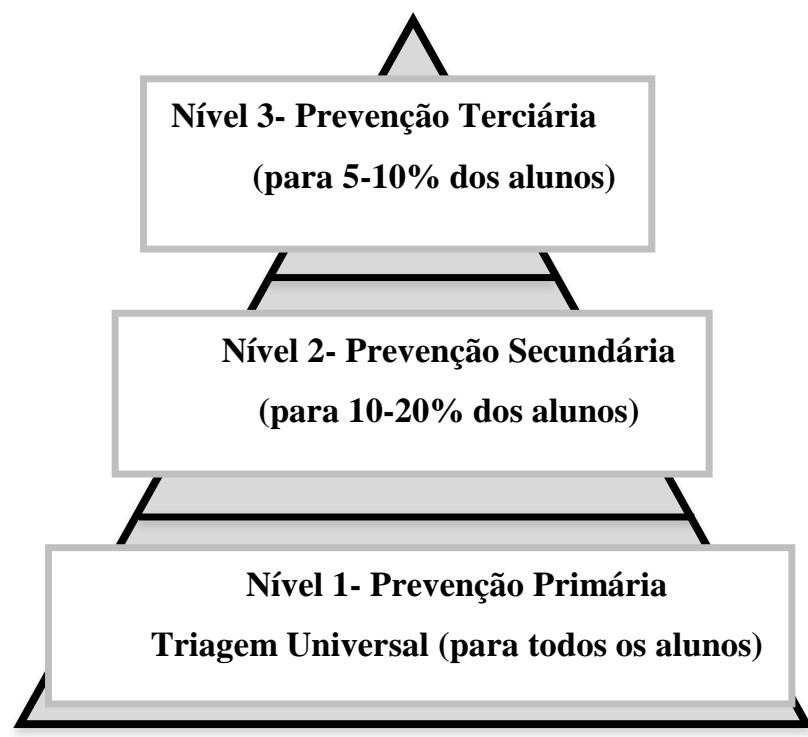

Fonte: Os autores (2018) Adaptado de VAZ; MARTINS; CORREIA (2018).

Destaca-se, no nível I do modelo, a triagem de todos os alunos (universal), com o objetivo de determinar precocemente quais são os que se encontram, ou podem vir a encontrar, em risco de terem baixos resultados de aprendizagem (FUCHS; FUCHS, 2008; CENTER RESPONSE TO INTERVENTION, 2012) e de, assim, precisarem de beneficiar de prevenção secundária (JOHNSON et al., 2006). A triagem universal constitui-se, assim, como o primeiro passo na detecção precoce de alunos em risco de evidenciarem dificuldades de aprendizagem específicas (HUGHES; DEXTER, 2013). É ainda seu objetivo, proporcionar a estes alunos uma intervenção precoce, no sentido de potenciar resultados positivos e de evitar a consolidação do insucesso académico ao longo da sua escolaridade, bem como as repercussões que este pode vir a ter durante toda a sua vida (FUCHS; FUCHS, 2008). Não existem critérios para selecionar apenas alguns alunos para participam nesta triagem, ou seja, todos participam pelo facto de frequentarem um estabelecimento de ensino. A triagem universal é feita três vezes no ano letivo, normalmente no início, no meio e no final, podendo incidir sobre uma ou mais áreas, como a leitura, a escrita, a matemática, o comportamento (FUCHS; FUCHS, 2008; HUGHES; DEXTER, 2013; JOHNSON 
et al., 2006). Durante estes três momentos a triagem assume diferentes finalidades (ver Tabela 1).

Tabela 1 - Frequência e finalidades da triagem universal

\begin{tabular}{c|l}
\hline \hline Momento do ano letivo & \multicolumn{1}{c}{ Finalidade } \\
\hline Início & - Identificar os alunos que apresentam um nível de realização \\
& escolar correspondente ao do início do ano letivo. \\
& - Identificar os alunos que estão com dificuldades e que \\
precisam de intervenção; ou seja os que não apresentam um \\
nível de realização escolar correspondente ao do início do ano \\
letivo.
\end{tabular}

Fonte: THE IRIS CENTER (2010).

Neste contexto deve ter-se em atenção a fidelidade da implementação da triagem, o nível cultural e linguístico dos alunos e os seus pontos fortes (CENTER RESPONSE TO INTERVENTION, 2012).

\section{A monitorização com base no currículo}

Para levar a cabo esta triagem pode recorrer-se à monitorização com base no currículo (MBC) (HUGHES; DEXTER, 2013, p. 3) que teve a sua origem nos anos setenta, na Universidade de Minnesota, onde Stanley Deno desenvolveu um projeto de investigação para determinar as suas questões técnicas e utilidade na planificação e na aprendizagem (FUCHS; STECKER, 2003). Representa uma forma de avaliação em condições estandardizadas, baseada no currículo nacional (STECKER; SÁENZ; LEMONS, 2007) e na utilização de provas de realização breve por todos os alunos nomeadamente, no caso da leitura, de provas orais e de compreensão (CENTER RESPONSE TO INTERVENTION, 2012). Deno et al. (2009) consideram que os alunos que apresentam resultados abaixo do percentil 20 são aqueles em risco académico. Consequentemente, ao considerarmos estes $20 \%$ de alunos, estamos a identificar aqueles que apresentam 
mais dificuldades e, na prática, a identificar um número de alunos considerado razoável para monitorizar individualmente. Assume-se, portanto, que a baixa realização relativamente a um ponto de referência, no início do ano letivo, é uma evidência de que um aluno está a falhar no nível 1 e necessita de prevenção secundária, ou de nível 2. Para a realização de $M B C$ na leitura existem dois procedimentos possíveis: a) MBC-Oral, que identifica a fluência através da contagem do número de palavras por minuto que o aluno lê corretamente, utilizando determinadas regras de cotação, entre as quais, descontar palavras nas quais o aluno hesita por mais de três segundos, palavras mal pronunciadas, substituições, omissões e reversões e não descontar em caso de repetição, inserções e autocorreção; b) MBC Maze (MBC-Maze), que identifica a compreensão da leitura (BROWN-CHIDSEY; DAVIS; MAYA, 2003; BUSCH; LEMBKE, 2005; DENO et al., 1989; SHINN; SHINN, 2002), mas que também nos fornece indicadores gerais da realização dos alunos nesta área, de acordo com Busch e Lembke (2005). Este procedimento implica que o aluno selecione a palavra correta entre três opções apresentadas. Em ambos os procedimentos, o número de palavras corretamente lidas ou selecionadas, numa amostra de leitura retirada de material curricular do ano do aluno e ainda não analisado nas aulas, constitui um indicador válido da proficiência da leitura.

\section{Os fatores de risco na leitura: o aluno e a família}

Fatores de risco não são nem causas nem explicações das dificuldades dos alunos na leitura, mas antes o resultado de correlações entre as suas características e essas mesmas dificuldades (MCCARDLE et al., 2001; SNOW; BURNS; GRIFFIN, 1998). A presença de fatores de risco não significa que os problemas na leitura venham necessariamente a verificar-se, sobretudo se estiver presente apenas um fator (NJCLD, 2007; SNOW et al., 1998; SPEECE et al., 2010), ou seja, é mais útil, por exemplo quando se estima a futura realização na leitura, a existência em simultâneo de um sistema que permita o despiste de vários fatores de risco (individuais, familiares e demográficos) (SNOW; BURNS; GRIFFIN, 1998). Uma vez identificados, eles não devem ser interpretados como fatores de inevitabilidade do insucesso, mas devem servir para alertar para o risco de aparecimento de dificuldades na leitura (SNOW; BURNS; GRIFFIN, 1998). 
Snow, Burns, Griffin. (1998), tendo por base a revisão da investigação efetuada por SCARBOROUGH (1998), apontam a existência de diferentes fatores de risco na leitura, mais concretamente de fatores que são intrínsecos ao aluno (a) e de fatores de risco relacionados com o contexto familiar (b), que se descrevem de seguida.

(a) Fatores de risco intrínsecos ao aluno

No contexto dos fatores de risco intrínsecos ao aluno Snow, Burns e Griffin (1998) referem-se a condições físicas e clínicas, à aquisição e desenvolvimento da linguagem e a preditores que podem estar presentes à entrada na escola, nomeadamente:

- Condições físicas e clínicas: Desde logo há condições perinatais como o baixo índice APGAR, a hospitalização durante mais de 24 horas em unidade de cuidados intensivos (NJCLD, 2007), o baixo peso à nascença/prematuridade (NJCLD, 2007; SNOW; BURNS; GRIFFIN, 1998), a síndrome alcoólica fetal, o envenenamento por chumbo, as graves carências alimentares ou a existência de uma condição psicopatológica grave (SNOW; BURNS; GRIFFIN, 1998) que se constituem como fatores de risco a considerar, pois sabe-se que tais situações podem deixar sequelas ao nível cerebral que, por sua vez, podem ter implicações ao nível dos processos de leitura. O mesmo se pode dizer da dificuldade intelectual, pois é sabido que crianças que a possuem têm performances muito pobres na leitura, podendo até nem conseguir ler (BURNS; GRIFFIN; SNOW, 1999; SNOW; BURNS; GRIFFIN, 1998). Também a otite média crónica, que pode ter como consequência a perda temporária de audição (NJCLD, 2007), e a diminuição da audição propriamente dita colocam uma criança em risco de experimentar problemas na leitura, mesmo que a perda auditiva não seja total e que haja uma boa capacidade de falar (BURNS; GRIFFIN; SNOW, 1999). De acordo com Jiménez et al. (1997) a aprendizagem da leitura em crianças com hipoacúsia é de elevada dificuldade. A pobreza de vocabulário, o escasso conhecimento da estrutura sintática, dificuldades de acesso ao código fonológico, a limitação da capacidade de antecipação, de inferência e de organização são fatores presentes em crianças com hipoacúsia e muito importantes na tarefa da leitura. Em muitos casos estes alunos conseguem dominar a mecânica da leitura sem, contudo, compreenderem o que leem (JIMÉNEZ et al., 1997). 
- Aquisição e desenvolvimento da linguagem: Segundo SNOW et al. (1998) diversos estudos indicam que as perturbações específicas da linguagem são identificadas em crianças com atrasos nas aprendizagens académicas. Entre 40 a $75 \%$ dos alunos do ensino pré-escolar com algum problema de linguagem apresentam posteriormente dificuldades na leitura (BURNS; GRIFFIN; SNOW, 1999). De acordo com SCARBOROUGH (1998) crianças com uma perturbação específica da linguagem correm um elevado risco de ter dificuldades em aprender a ler. As dificuldades articulatórias, muitas vezes vistas como ficando a deverse a uma certa infantilidade, mas cuja existência se prolonga para além da idade em que são usuais (aos cinco/seis anos estas dificuldades a existirem devem ser escassas) podem estar na base de uma dificuldade grave na leitura como é o caso da dislexia (SHAYWITZ, 2008).

Snow, Burns e Griffin (1998) referem o estudo de Shapiro et al. (1990) como o único que analisa a previsão do tipo de realização na leitura a partir das diferenças na aquisição e desenvolvimento da linguagem em crianças. Shapiro et al. (1990) verificaram que crianças com um atraso no desenvolvimento da linguagem apresentam maior risco de virem a ter dificuldades na leitura. Segundo Burns, Griffin e Davis (1999) o desenvolvimento da linguagem durante os primeiros anos está estreitamente relacionado com a aprendizagem da leitura, mais concretamente no que respeita à linguagem expressiva que está fortemente relacionada com a realização na leitura nos anos escolares. Também Shaywitz (2008), referindo-se à dislexia, refere que um atraso na linguagem pode ser um primeiro indicador deste tipo de Necessidade Educativa Especial. Ao invés do que é esperado, as crianças em risco de apresentarem dislexia podem começar a dizer as primeiras palavras por volta dos 15 meses e a fazer uso de pequenos enunciados depois dos dois anos (o esperado seria que estas aprendizagens tivessem lugar aos 12 e aos 18 meses, respetivamente). Muitas vezes, dado aparentemente não se tratar de um atraso preocupante, é desvalorizado ou atribuído à história familiar. Mas, efetivamente, um atraso desde género, ainda que aparentemente desvalorizável, numa família com historial de dislexia pode ser um alerta para o aparecimento de um problema ao nível da leitura. Todavia, nem todas as crianças com dislexia apresentam este atraso ou ele pode ser tão ténue que passa despercebido (SHAYWITZ, 2008). 
Apesar de serem problemáticas distintas, é frequente as dificuldades de a leitura ocorrerem em simultâneo com o défice de atenção/hiperatividade (NJCLD, 2007; SNOW; BURNS, GRIFFIN, 1998), situação que parece poder justificar-se com as palavras de García (2001) quando refere que as crianças com este tipo de perturbação revelam dificuldades em captar, organizar e processar a informação sensorial e em expressá-la depois. Igualmente Mccardle et al. (2001), referindo-se ao deficit de atenção, salientam que mesmo possuindo boas competências cognitivas e linguísticas, um aluno falhará na leitura se não estiver atento, quer durante o processo de aprendizagem, quer durante a prática da leitura.

- Preditores à entrada na escola: O seguinte conjunto de preditores pode estar presente e ser avaliado à entrada na escola (SNOW; BURNS; GRIFFIN, 1998): proficiência adquirida na linguagem (memória verbal, competências lexical e sintática, consciência fonológica); conhecimento adquirido ao nível da literacia (identificação de letras, conceitos de impressão).

Para o processo de leitura e para a aprendizagem em geral é importante reter a informação verbal na memória de trabalho, situação que faz com que se espere que a memória verbal constitua também um preditor da futura realização na leitura, como encontrado na revisão de Scarborough (1998). Mais concretamente a repetição de frases e de uma breve história em crianças no jardim de infância surgem na revisão de Scarborough (1998) como preditores fiáveis. O mesmo acontece com a nomeação de vocabulário (SCARBOROUGH, 1998). Os resultados dos estudos revistos em Scarborough (1998), que incluem por exemplo medidas que avaliam a nomeação de objetos, remetem para o facto de esta capacidade ser um preditor fiável da capacidade de leitura futura (SNOW; BURNS; GRIFFIN, 1998). Como preditores surgem também a compreensão de frases e a linguagem expressiva (produção de linguagem) (SNOW; BURNS; GRIFFIN, 1998), na medida em que a compreensão de um texto está dependente da linguagem oral do leitor, no que respeita à compreensão dos significados das palavras que são identificadas e às relações sintáticas e semânticas entre elas (SCARBOROUGH, 1998). Também a consciência fonológica surge como preditor da realização futura na leitura, na revisão de Scarborough (1998). Segundo o National Early Literacy Panel - (NELP, 2008) é forte a evidência que remete para a importância da consciência fonológica enquanto preditor da realização na leitura. 
Neste contexto, Mccardle et al. (2001) trazem à discussão um modelo que na tentativa de contribuir para a compreensão das dificuldades na leitura, coloca ênfase na existência de um deficit fonológico central, com repercussão ao nível da consciência fonológica, da descoberta do princípio alfabético e ao nível da aprendizagem da descodificação (STANOVICH; SIEGEL, 1994). Numa apresentação mais detalhada deste modelo Scarborough (2005) remete para a existência de um atraso no desenvolvimento do sistema fonológico nos primeiros três anos de vida, que conduz a uma perda na consciência fonológica que se manifesta entre os quatro e os cinco/seis anos de idade e que tem repercussões, nos primeiros anos de escolaridade, ao nível da precisão e da velocidade no reconhecimento de palavras, da descodificação e da ortografia. Estes deficits traduzem-se, consequentemente, à medida que a idade e a escolaridade avançam, em desprovimentos secundários ao nível da compreensão e numa reduzida experiência de leitura, que por sua vez conduzirão a deficits terciários ao nível do vocabulário, do QI e do conhecimento em geral. Ainda que a influência da consciência fonológica na leitura seja indiscutível (MCCARDLE et al., 2001) trata-se, contudo, de acordo com Mccardle et al. (2001), de um modelo que não representa tudo o que já se conhece acerca dos problemas de leitura, e que não estabelece relações por exemplo com perturbações específicas da linguagem (SCARBOROUGH, 2005). Na tentativa de expandir a ideia central deste modelo outros foram surgindo.

Num outro modelo apresentado em Mccardle et al. (2001) o défice fonológico e consequentemente a consciência fonológica bem como outros aspetos relacionados com o processamento do discurso, surgem como manifestação de uma deficiência no processamento auditivo. No entanto, também esta conceção é insuficiente para explicar os problemas na leitura, não sendo sequer defendido pela maioria da comunidade científica (MCCARDLE et al., 2001).

Num terceiro modelo é destacado a uma dupla perda, quer ao nível fonológico quer ao nível da velocidade de processamento, sendo que o primeiro se traduz em problemas ao nível da consciência fonológica e o segundo numa maior lentidão em tarefas de nomeação. De acordo com este modelo os problemas na leitura podem resultar de um destes deficits ou de ambos, sendo no segundo caso mais grave (MCCARDLE et al., 2001). Contudo, a velocidade de nomeação lenta é vista pelos proponentes do modelo de carência fonológica 
central como uma outra manifestação deste mesmo deficit não como um fator separado (MCCARDLE et al., 2001).

Num quarto modelo a ênfase é colocada em dificuldades ao nível da linguagem, iniciadas ainda durante os anos do pré-escolar, que conduzem a problemas ao nível fonológico, por um lado, com repercussões ao nível do reconhecimento de palavras, e aos níveis sintático e semântico por outro, com repercussões ao nível da compreensão (MCCARDLE et al., 2001).

Ainda no contexto dos preditores à entrada na escola Snow et al. (1998) referem-se ao conhecimento adquirido ao nível da literacia, através da exposição, ainda antes de aprenderem a ler, a informação acerca dos objetivos da leitura, dos seus mecanismos e das competências necessárias na leitura. Referem a preparação para a leitura, que inclui nomeadamente a capacidade de identificar letras e os conceitos de impressão (conhecimento sobre a forma como o material impresso e não somente as letras, pode ser usado), enquanto preditores que foram estudados no sentido de se saber se as diferenças encontradas nestas capacidades predizem diferenças na leitura. De acordo com Scarborough (1998) e Davis, Lindo e Compton (2007) a identificação de letras (número de letras que uma criança é capaz de nomear, no jardim de infância), surge como o mais forte preditor no contexto dos preditores que constituem as capacidades que no seu conjunto permitem dizer que um aluno está preparado para ler, conhecimento este corroborado também pelos resultados de Clemens et al. (2012).

(b) Fatores de risco parentais e contextuais

No contexto dos fatores de risco relacionados com os pais e o contexto, Snow, Burns; Griffin (1998) destacam os seguintes:

- História familiar de problemas na leitura;

- Ambiente de literacia em casa: valor que os pais dão à leitura, pressão para a realização, disponibilidade e uso instrumental de materiais de leitura, leitura com as crianças, oportunidades de interação verbal;

- Uso de outra língua em casa além da falada no país onde vivem;

- Uso de um dialeto em casa;

- Estatuto socioeconómico. 
De acordo com Snow, Burns e Griffin (1998) e com o National Joint Committee on Learning Disabilities (2007) tendo em conta décadas de investigação, a história familiar de problemas na leitura parece aumentar o risco de estas surgirem. Scarborough (1998) verificou na sua revisão da investigação acerca dos preditores de risco, que a existência de dislexia num familiar coloca uma criança em risco elevado de vir a ser identificada como tendo dislexia. Segundo Shaywitz (2008), de acordo com a investigação recente a dislexia é transmissível geneticamente. De facto, a dislexia pode manifestar-se em vários elementos de uma família, pelo que um aluno que tem um dos pais ou um irmão com dislexia, tem aumentada a probabilidade de ter este tipo de NEE (SHAYWITZ, 2008). Contudo, a existência de dislexia por parte dos pais não significa necessariamente que esta também será experienciada pelos filhos (BURNS et al., 1999; SHAYWITZ, 2008).

Também o conhecimento do ambiente de literacia em casa pode fornecer indicação sobre o risco de uma criança nele inserido poder vir a ter problemas na leitura (SCARBOROUGH, 1998; SNOW; BURNS; GRIFFIN, 1998). Assim, importa perceber o valor que os pais dão à leitura, demonstrado por exemplo através da sua própria leitura e do encorajamento/motivação dos seus educandos para a mesma. É importante saber também qual o apoio dado por parte dos pais à leitura dos seus educandos, bem como se aqueles respondem positivamente à iniciativa destes e ao seu interesse para ler. A disponibilidade e o uso de materiais de leitura podem influenciar também o desenvolvimento desta, na medida em que se existirem, nomeadamente livros infantis, será mais provável a existência de experiências de literacia em casa. O mesmo acontece relativamente à prática de leitura com as crianças, podendo esta assumir diferentes formas, nomeadamente a leitura dos pais para as crianças e a leitura das crianças para os pais, com o apoio destes quando necessário, situações que podem acontecer por exemplo à noite antes de dormir (PUT READING FIRST, 2000). Scarborough (1998) verificou que a quantidade de leitura dos pais em casa surge como um preditor fraco, mas a disponibilidade de materiais de leitura em casa e a frequência de bibliotecas apresentam uma correlação fiável com a realização futura na leitura. A quantidade de interação verbal no seio familiar tem também influência no desenvolvimento da leitura, sendo que uma baixa interação verbal no seio familiar funciona como um fator de risco. 
Também o The Partnership For Reading (PUT READING FIRST, 2000) aponta a importância da interação verbal em casa, nomeadamente na hora das refeições, justificando que as crianças aprendem palavras com maior facilidade quando as ouvem muitas vezes.

A existência de uma outra língua no contexto familiar constitui também um fator de risco de insucesso na leitura (SNOW; BURNS; GRIFFIN, 1998). De acordo com Burns, Griffin e Snow (1999) nos EUA os alunos hispânicos são alunos em risco de terem dificuldades na leitura, sendo duas vezes mais provável que tenham uma performance na leitura inferior ao esperado para a sua idade do que os alunos brancos não hispânicos. Acresce o facto de muitos destes alunos terem pais com baixa escolaridade, pertencerem a famílias com rendimentos económicos baixos, viverem em comunidades também com baixas possibilidades económicas e frequentarem escolas cujos alunos apresentam baixos resultados académicos. Snow; Burns e Griffin (1998) refere que existe uma dificuldade associada quando se pretende avaliar o risco relacionado com a baixa proficiência na língua que é usada na escola (em virtude da existência de outra língua em casa). Esta dificuldade prende-se mais concretamente com a existência de diferenças culturais e linguísticas que podem acarretar outros fatores de risco, como por exemplo: a) os alunos em questão pertencerem a minorias que residem em comunidades com muitas famílias; b) a existência de diferenças culturais entre a família e a escola (no que respeita por exemplo a valores e expectativas), de fatores sociopolíticos relacionados com a discriminação e com a falta de oportunidades.

O uso de um dialeto em casa, diferente daquele que é ensinado na escola, pode estar na base do aparecimento de problemas na aprendizagem da leitura (BURNS; GRIFFIN; SNOW, 1999). Quando confrontado com a necessidade de fazer corresponder letras a sons numa palavra, o aluno que usa um dialeto pode apresentar dificuldades (SNOW; BURNS; GRIFFIN, 1998). Por vezes determinados dialetos são confundidos com a utilização de um Inglês incorreto (BURNS; GRIFFIN; SNOW, 1999; SNOW; BURNS; GRIFFIN, 1998), relacionados por exemplo com um atraso no desenvolvimento da linguagem, aprendizagem pobre, articulação descuidada, e até insolência propositada. Pode acontecer inclusivamente que professores menos sensíveis à existência de dialetos desenvolvam perceções negativas e expectativas baixas relativamente à realização destes 
alunos e assim baixem o nível de exigência para com os mesmos (SNOW; BURNS; GRIFFIN, 1998).

Apesar de famílias com estatuto socioeconómico baixo não terem necessariamente menos educação (SNOW; BURNS; GRIFFIN, 1998) tende a acontecer que crianças nelas inseridas têm pais com menor instrução (BURNS; GRIFFIN; SNOW, 1999) e vivem muitas vezes em comunidades caraterizadas pelas fracas possibilidades económicas, em que aparecem comprometidos aspectos como a nutrição, o acesso a serviços de saúde nomeadamente a cuidados pré-natais e pediátricos (SCARBOROUGH, 1998; SNOW; BURNS; GRIFFIN, 1998) - e a qualidade das escolas, circunstâncias que podem prejudicar o desenvolvimento das crianças (SCARBOROUGH, 1998). Cruzam-se aqui, portanto, os fatores de risco relacionados mais especificamente com a comunidade e com a escola. Contudo, estar inserido numa família com baixos rendimentos económicos, não significa necessariamente que exista maior risco de aparecerem problemas na leitura se forem proporcionados o ensino e o apoio necessários o que faz com que a pobreza e/ou a inserção em famílias com baixos rendimentos económicos não possa ser visto por si só, como um indicador de risco (BURNS; GRIFFIN; SNOW, 1999).

No contexto anteriormente descrito, nomeadamente de triagem universal, efetuouse uma pesquisa que teve como finalidade caraterizar alunos identificados em risco na leitura. Assim foram dois os seus objetivos: 1) comparar a compreensão leitora (realização e crescimento) de alunos do 30 ano de escolaridade, segundo a variável risco; 2) identificar nos alunos em risco fatores que lhe são intrínsecos, parentais e contextuais.

\section{Método}

\section{Participantes}

Participaram nessa pesquisa 82 alunos com oito anos de idade (47 raparigas e 35 rapazes), distribuídos por seis turmas diferentes, correspondentes à população que frequenta o 3. ano do 1. Ciclo do Ensino Básico de um Agrupamento de Escolas situado em Trás-os-Montes (Portugal). Nenhum dos alunos tinha Necessidades Educativas Especiais identificadas e nenhum era apoiado por serviços de educação especial.

Após três momentos de triagem realizados no ano letivo foi possível observar que 10 alunos se mantiveram em risco nos três momentos, e que 53 nunca apareceram em risco 
na compreensão da leitura. Os restantes 19 são alunos que apresentaram risco em algum momento de triagem. Dos 10 alunos que ficaram em risco nos três momentos de triagem, sete foram posteriormente caraterizados no que respeita aos fatores de risco neles presentes, tratando-se mais concretamente de seis raparigas e um rapaz.

\section{Instrumentos de coleta de dados}

Nessa pesquisa, para a coleta de dados, usaram-se dois instrumentos:

1) Prova de monitorização com base no currículo para a compreensão da leitura prova de MBC-Maze: A prova foi elaborada para essa pesquisa, iniciando-se em primeiro lugar um trabalho de busca de textos de manuais escolares do 30 ano de escolaridade, para a seguir selecionar o que, pelas suas caraterísticas, melhor se adequasse às regras de elaboração da mesma. Desde logo foram consultados diversos manuais, diferentes do adotado pelo agrupamento, no sentido de restringir a probabilidade de poderem estar a ser objeto de análise textos que já tivessem sido trabalhados na aula ou cujo trabalho estivesse programado por algum dos professores. Neste sentido, após selecionado o texto e havendo a indicação de que este não havia sido trabalhado em nenhuma das turmas, decidiu-se pela elaboração da prova com base no mesmo e acordou-se com os docentes que estes não o trabalhariam nas suas aulas. Teve-se também o cuidado de escolher um texto que não fosse alusivo a temas específicos, como por exemplo as estações do ano ou quadras festivas.

Uma vez escolhido o texto procedeu-se à elaboração da prova, respeitando-se as seguintes regras: (1) Deixar intacta a primeira frase (BUSCH; LEMBKE, 2005; SHINN; SHINN, 2002); (2) Apagar, a partir da primeira frase, cada sétima palavra e no lugar dela colocar três palavras: a palavra original que pertence ao texto (palavra correta) e duas outras que não fazem sentido no texto (distratores) (BUSCH; LEMBKE, 2005; SHINN; SHINN, 2002); (3) Ter a certeza de que nenhum dos distratores faz sentido no texto. Apenas a palavra correta pode fazer sentido no texto (BUSCH; LEMBKE, 2005).

No que respeita concretamente aos distratores, tiveram-se em conta as regras que se seguem: (1) Não escolher os distratores ao acaso. Um deles deve ser do mesmo tipo da palavra correta (nome, verbo, advérbio) e o outro não do mesmo tipo, mas uma palavra retirada aleatoriamente do texto (SHINN; SHINN, 2002); (2) Escolher distratores 
do mesmo comprimento que a palavra que pertence ao texto (palavra correta) (BUSCH; LEMBKE, 2005); (3) Selecionar distratores que não rimem com a palavra correta (Busch e Lembke, 2005); (4) Escolher distratores que não comecem pela mesma letra da palavra correta (BUSCH; LEMBKE, 2005).

Foram consideradas, ainda, algumas sugestões adicionais segundo Busch e Lembke (2005): (1) Sublinhar e colocar em negrito as três palavras (a correta e os distratores); (2) Não deixar separadas as três palavras no final das linhas do texto; (3) Passar para a palavra anterior ou para a palavra seguinte quando a sétima palavra for um nome próprio; (4) Variar a localização da palavra correta relativamente aos distratores; (5) Escrever as três palavras com letra maiúscula, quando a sétima palavra for a primeira palavra de uma frase.

A prova é constituída por uma folha de rosto onde o aluno tem de colocar os seus dados pessoais (nome e idade) bem como a data da sua realização, e que contém ainda um exemplo constituído por três frases de treino, em que cada uma tem uma situação de escolha da palavra que faz sentido na frase. A esta folha de rosto segue-se o texto onde surgem 33 situações em que o aluno tem de selecionar a palavra que faz sentido. É, portanto, igual a 33 o resultado máximo que um aluno pode atingir nesta prova Maze.

Foi definido o limite de tempo de três minutos (BUSCH; LEMBKE, 2005; GRANEY et al., 2010; RICHARDSON; HAWKEN; KIRCHER, 2012; SHIN; DENO; ESPIN, 2000; SHINN; SHINN, 2002) e optou-se pela realização coletiva em cada turma (BUSCH; LEMBKE, 2005; SHINN; SHINN, 2002). As provas foram cotadas e a pontuação de cada aluno correspondeu ao número de seleções corretas até o aluno apresentar três erros consecutivos (PATRÃO, 2010).

É importante realçar que a adequação técnica deste tipo de provas é suportada por um vasto caudal de investigação que tem vindo a tornar claro que se trata de um instrumento fiável e válido para a avaliação da competência de leitura (DENO et al., 2009; ESPIN et al., 2010; MERCER et al., 2012; PIERCE; MACMASTER; DENO, 2010; TICHÁ; ESPIN; WAYMAN, 2009).

2) Grelha para a identificação de fatores de risco na leitura: Depois de um trabalho prévio de pesquisa bibliográfica e de revisão da literatura nas bases de dados eletrónicas PsycARTICLES, EBSCO Electronic Journals Service, Scielo e Redalyc, com as palavras-chave 
risco na leitura, fatores de risco na leitura, preditores de risco na leitura, reading risk, risk factors, risk predictors, elaborou-se um instrumento de recolha de dados, que se constitui como um documento de apoio aos professores na identificação de fatores de risco na leitura, nomeadamente através da recolha de informação relativa aos fatores de risco indicados por Snow, Burns e Griffin (1998). Esta grelha contempla três pontos essenciais.

O primeiro ponto diz respeito a informações sobre o agregado familiar em que o aluno se insere, nomeadamente relativas ao grau de parentesco que os diferentes elementos têm com o aluno, às respetivas habilitações literárias e profissões (CORREIA, 2013).

O segundo ponto é relativo aos fatores de risco intrínseco ao aluno, mais concretamente a condições físicas e clínicas e aos preditores à entrada na escola também referidos por Snow, Burns e Griffin (1998).

O terceiro ponto é dedicado aos fatores de risco parentais e contextuais, procurandose averiguar se há na família situações de dificuldades na leitura, e conhecer aspetos que dizem respeito ao ambiente de literacia em casa nomeadamente à existência ou não de práticas de leitura por parte dos pais na presença dos filhos, à existência ou não de pressão/motivação por parte dos pais para que os filhos leiam, à disponibilidade de materiais de leitura, à existência de hábitos de leitura com as crianças e à compreensão da forma como se concretizam ou não oportunidades de interação verbal no seio familiar. Ainda neste terceiro ponto procura-se saber se em casa é falada outra língua, além do Português, ou um dialeto, e conhecer o estatuto socioeconómico da família do aluno.

É ainda dedicado espaço para a anotação de observações que no decorrer da recolha de dados possam emergir como pertinentes.

Esta grelha deve ser preenchida pelo professor do ensino regular, junto dos pais/encarregado de educação do aluno (VAZ, 2015).

\section{Procedimentos de coleta de dados}

Tal como na pesquisa realizada por Vaz, Martins e Correia (2016), num primeiro momento foram solicitadas as necessárias autorizações (à direção do agrupamento de escolas, aos professores e aos encarregados de educação). Uma vez obtidas e depois de se 
consultarem os alunos no sentido de manifestarem a sua concordância relativamente à participação na pesquisa, calendarizaram-se os três momentos de triagem universal para a coleta de dados. Desse modo, realizou-se na fase inicial do ano letivo (outono) um primeiro momento de coleta com a prova de MBC-Maze, no meio do ano letivo (inverno) uma segunda coleta e no final do ano letivo (primavera) uma terceira coleta. Nos diferentes momentos de coleta de dados garantiu-se a fiabilidade da administração das provas através da comprovação da manutenção dos procedimentos concretos respeitantes às instruções fornecidas aos alunos, aos exemplos de treino e ao limite de tempo. Neste sentido, e para garantir que todos os procedimentos foram realizados e repetidos de igual modo, nos diferentes momentos, o professor responsável por cada turma preencheu uma grelha de validação para a prova Maze (VAZ, 2015).

Depois de conhecidos os alunos que na prova de $M B C$-Maze ficaram em risco nos três momentos da triagem, isto é, os que se encontravam abaixo do percentil 20 , foi obtida autorização por parte dos respetivos pais/encarregado de educação para o preenchimento do instrumento "Grelha para a identificação de fatores de risco na leitura". Reuniu-se com cada um, em datas previamente agendadas, numa sala do agrupamento/escola destinada ao efeito, com a necessária tranquilidade e sem a presença de outros indivíduos. Durante esta reunião esclareceram-se, em primeiro lugar, dúvidas que ainda existissem sobre as informações que iriam ser pedidas e solicitou-se o seu consentimento escrito para a utilização, ainda que anónima, das informações fornecidas para a sua utilização na investigação. Estabeleceu-se depois um diálogo cordial em que os pais/encarregado de educação foram sendo questionados relativamente aos itens que constituem a grelha em questão e se foram registando, na sua presença, as informações consideradas pertinentes.

\section{Resultados}

\section{Resultados relativos ao nível e taxa de crescimento na compreensão da leitura}

Apresentam-se de seguida (ver Tabela 2) as medidas estatísticas de tendência central - Média (M), Moda (Mo) e Mediana (Me) -, os valores mínimo e máximo e uma medida de dispersão, o Desvio Padrão (DP), obtidos pelos sete alunos que estiveram em risco durante todo o ano letivo e pelos alunos que nunca estiveram em risco no variável nível da compreensão leitora. 
Tabela 2 - Descrição estatística dos resultados na prova Maze dos alunos em risco durante todo $\mathrm{o}$ ano letivo e dos alunos que nunca estiveram em risco

\begin{tabular}{c|c|c|c|c|c|c|c}
\hline \hline & $n$ & $M$ & $D P$ & $M o$ & $M e$ & Mínimo & Máximo \\
\hline outono & & & & & & & \\
Em risco & 7 & 3.43 & 2.070 & $1^{*}$ & 4.00 & 1 & 6 \\
Sem risco & 53 & 11.25 & 3.322 & 11 & 11.00 & 7 & 22 \\
inverno & & & & & & & \\
Em risco & 7 & 5.14 & 3.436 & $0^{*}$ & 6.00 & 0 & 9 \\
Sem risco & 53 & 17.68 & 5.121 & 16 & 16.00 & 11 & 31 \\
primavera & & & & & & & \\
Em risco & 7 & 9.00 & 1.528 & $7^{*}$ & 9.00 & 7 & 11 \\
Sem risco & 53 & 18.91 & 4.732 & 18 & 18.00 & 12 & 32 \\
\hline \hline
\end{tabular}

*Existem múltiplas modas. É apresentado o valor mais baixo.

Fonte: VAZ (2015).

Através da análise da Tabela 2 constata-se que em ambos os grupos de alunos, na Prova de $M B C-M a z e$, o valor médio de respostas corretas aumenta de monitorização para monitorização. Destaca-se, no entanto, que na monitorização do outono e na do inverno, o resultado alcançado pelos alunos sem risco é maior do que o triplo do resultado alcançado pelos alunos em risco, sendo na monitorização da primavera cerca de duas vezes mais elevado.

Efetuou-se o cálculo do valor médio das taxas de crescimento semanal obtidas pelos sete alunos que se mantiveram em risco no decorrer do ano letivo e por aqueles que nunca apresentaram risco na variável nível de compreensão leitora, nos seguintes intervalos de tempo: desde uma fase inicial do ano letivo (outono) até a uma fase intermédia do mesmo (inverno); desde a mesma fase intermédia até a uma fase final do ano letivo (primavera); desde a mesma fase inicial referida anteriormente (outono) até à mesma fase final do ano (primavera). Para o cálculo destas taxas utilizou-se a fórmula proposta Brown e Skow (2009) que consiste em encontrar a diferença entre o resultado na segunda monitorização e o resultado na primeira monitorização e em calcular o quociente entre essa diferença e o número de semanas entre as duas monitorizações.

Observando a Tabela 3 verifica-se que os resultados monstram que no intervalo de tempo que decorre entre a fase inicial do ano letivo (outono) e o período intermédio deste mesmo ano (inverno) são os alunos que nunca apresentaram risco que crescem mais na leitura. Já no período que vai desde o inverno até à fase final do ano (primavera) é maior o crescimento nos alunos que ficaram sempre em risco. Tendo em conta as três monitorizações efetuadas no decurso do ano letivo, os alunos de risco crescem mais entre 
a fase intermédia do ano (inverno) e a fase final (primavera) do que no intervalo entre a fase inicial do ano (outono) e o meio do ano (inverno). Verifica-se exatamente o inverso nos alunos que nunca evidenciaram risco. Considerando a totalidade do ano letivo, isto é, o crescimento entre uma fase inicial do ano (outono) e a fase final do ano (primavera), observa-se que quem apresenta maior crescimento na leitura são os alunos que em nenhum momento evidenciaram risco.

Tabela 3 - Taxas médias de crescimento entre diferentes monitorizações no ano letivo, para os alunos em risco durante todo o ano letivo e os alunos que nunca estiveram em risco

\begin{tabular}{c|c}
\hline \hline & \multicolumn{2}{|c}{ Taxa média de crescimento } \\
\hline outono-inverno & .13 \\
Em risco & .49 \\
Sem risco & \\
inverno-primavera & .39 \\
Em risco & .13 \\
Sem risco & \\
outono-primavera & .23 \\
Em risco & .32 \\
Sem risco
\end{tabular}

Fonte: VAZ (2015).

É importante salientar que quando se considera o período que vai desde o outono até ao inverno os alunos sem risco crescem cerca de três vezes mais quando comparados com os alunos que se mantiveram sempre em risco. Tal situação altera-se quando se considera o intervalo que vai desde o inverno até a primavera, pois nesse os alunos que se mantiveram sempre em risco crescem o triplo dos alunos sem risco. Assim, podemos pensar, como sugerido por Vaz, Martins e Correia (2016), que pode ter havido maior atenção do professor entre o inverno e a primavera, direcionada aos alunos que entre o outono e o inverno mostraram problemas na leitura. Os alunos que nunca mostraram qualquer risco, sendo bons leitores e estando no 3 ㅇ ano de escolaridade crescem cada vez menos porque na verdade eles já sabem ler, apenas vão aperfeiçoando, a partir daqui, a sua leitura.

\section{Resultados Relativos aos fatores intrínsecos aos alunos, aos pais e ao contexto}

Apresentam-se de seguida os fatores de risco presentes nos sete alunos que foram identificados em risco nas diferentes monitorizações realizadas no ano letivo, no contexto do sistema de triagem implementado. Faz-se uma apresentação quantitativa destacandose num primeiro momento os fatores de risco intrínsecos aos alunos e num segundo 
momento os fatores de risco relacionados com os pais e o contexto. Importa referir que, dada a impossibilidade de avaliar, no âmbito deste estudo, os preditores à entrada na escola e o estatuto socioeconómico, não são aqui apresentados quaisquer resultados relativos a estes fatores de risco.

Relativamente às condições físicas e clínicas salienta-se o facto de, dos sete alunos em risco no ano letivo, cinco apresentarem pelo menos uma condição física e clínica. Destes, quatro alunos têm Perturbações da Linguagem/Perturbações da Fala e um apresenta Deficit de Atenção. Verifica-se a existência de atraso no desenvolvimento da linguagem, em três alunos e de problemas de articulação em três alunos também. Interessa contudo sublinhar que dois dos alunos têm simultaneamente atraso no desenvolvimento da linguagem e problemas de articulação.

No que respeita aos fatores de risco relacionados com os pais e o contexto familiar, mais concretamente à existência, na família, de problemas na leitura constata-se que em nenhum dos sete alunos há história familiar de problemas na leitura. Quanto ao ambiente de literacia em casa, no que respeita às práticas de leitura dos pais na presença dos filhos, apenas no caso de um aluno, há pelo menos um dos seus progenitores que tem o hábito de ler na sua presença. Relativamente à pressão/motivação para a leitura, a maioria dos encarregados de educação (seis encarregados de educação) refere que pressiona e motiva os seus educandos para a leitura, solicitando que leiam textos do livro de língua portuguesa e/ou livros de histórias e chamando a atenção para a necessidade de saberem ler bem. Em todos os lares existem livros infantis à disposição dos alunos. O hábito de leitura com as crianças existe em todos os agregados familiares, destacando-se maioritariamente (cinco agregados familiares) a leitura da criança para pelo menos um dos progenitores. Apenas num agregado familiar há o hábito de pelo menos um progenitor ler para a criança e num outro agregado o hábito de pelo menos um dos progenitores ler para a criança e a criança ler para o mesmo a seguir. Contudo importa perceber que, se por um lado a existência de hábitos de leitura com as crianças em todos os agregados familiares remete para uma situação desejável, a verdade é que a concretização destes hábitos de leitura se traduz, maioritariamente, na leitura da criança para o adulto, havendo apenas num dos agregados a situação mais desejável que consiste na leitura de pelo menos um dos progenitores para a criança e, em seguida, desta para o progenitor. Relativamente à existência de oportunidades de interação verbal elas existem para todos os alunos ocorrendo sobretudo no dia-a-dia, quando os alunos em casa 
contam aos pais o que fizeram na escola. Em nenhum dos agregados familiares se usa outra língua em casa além do Português, ou se usa um dialeto.

Sublinha-se que os sete alunos que estiveram em risco ao longo do ano letivo apresentam entre um e três fatores de risco, sendo de registar que seis destes alunos apresentam pelo menos dois fatores de risco (ver Tabela 4).

Tabela 4 - Fatores de risco presentes em cada aluno

\begin{tabular}{|c|c|c|}
\hline \multirow{2}{*}{ Alunos } & \multicolumn{2}{|c|}{ Fatores de Risco } \\
\hline & Intrínsecos ao aluno & Parentais e contextuais \\
\hline 1 & & $\begin{array}{l}\text { - Ausência de práticas de leitura dos } \\
\text { pais na presença do aluno; } \\
\text { - Não é pressionado/motivado para a } \\
\text { leitura, no seio familiar; }\end{array}$ \\
\hline 2 & & $\begin{array}{l}\text { - Ausência de práticas de leitura dos pais } \\
\text { na presença dos filhos. }\end{array}$ \\
\hline 3 & $\begin{array}{l}\text { - Problemas de articulação; } \\
\text { - Atraso no desenvolvimento da } \\
\text { linguagem; }\end{array}$ & \\
\hline 4 & $\begin{array}{l}\text { - Problemas de articulação; } \\
\text { - Atraso no desenvolvimento da } \\
\text { linguagem; }\end{array}$ & $\begin{array}{l}\text { - Ausência de práticas de leitura dos } \\
\text { pais na presença do aluno; }\end{array}$ \\
\hline 5 & - Deficit de Atenção; & $\begin{array}{l}\text { - Ausência de práticas de leitura dos } \\
\text { pais na presença do aluno; }\end{array}$ \\
\hline 6 & $\begin{array}{l}\text { - Atraso no desenvolvimento da } \\
\text { linguagem; }\end{array}$ & $\begin{array}{l}\text { - Ausência de práticas de leitura dos } \\
\text { pais na presença do aluno; }\end{array}$ \\
\hline 7 & - Problemas de articulação & $\begin{array}{l}\text { - Ausência de práticas de leitura dos } \\
\text { pais na presença do aluno; }\end{array}$ \\
\hline
\end{tabular}

Fonte: VAZ (2015).

Importa acrescentar que dos sete alunos, quatro têm simultaneamente fatores de risco intrínsecos ao aluno e fatores de risco parentais e contextuais, um tem apenas fatores de risco intrínsecos ao aluno e dois apresentam apenas fatores de risco parentais e contextuais.

\section{Conclusões}

Com esta pesquisa procurou-se chamar a atenção para os alunos que nos primeiros anos de escola se deparam com problemas na aprendizagem da leitura. A partir daqui buscou-se um conhecimento mais aprofundado sobre estes alunos, quer no que respeita à sua performance na leitura, nomeadamente ao nível da compreensão, quer no que 
respeita aos fatores de risco que neles possam estar presentes. Desta forma emergiram da pesquisa efetuada, três conclusões principais:

a) Na fase inicial do ano letivo, os resultados médios para o nível de compreensão da leitura dos alunos que nunca mostraram risco são superiores ao triplo dos resultados médios daqueles que ficaram em risco durante todo o ano letivo, e no final do ano letivo é sensivelmente duas vezes mais elevado. Esta situação foi encontrada também por Deno et al. (2009) que encontraram no início do ano letivo um resultado mais do que três vezes mais elevado nos alunos sem risco relativamente aos alunos em risco e nas restantes monitorizações (inverno, primavera), resultados nos alunos sem risco que são mais elevados do que o dobro daqueles apresentados pelos alunos em risco. Também é relevante salientar que é no outono que os alunos em risco distam menos dos colegas que nunca mostraram risco e no inverno e na primavera que estão mais distantes destes colegas. É, aliás, no tempo que decorre do outono até ao inverno que eles se distanciam mais. Tal como indicado por Vaz, Martins e Correia (2016) esta situação deve conduzir a uma reflexão em torno da urgência de uma atuação atempada por parte do sistema de ensino, pois caso este não atue numa fase inicial do ano letivo os alunos em risco distanciam-se cada vez mais dos colegas sem risco, sendo de considerar as respetivas repercussões desta situação no sucesso académico destes alunos. Torna-se assim evidente que é necessário atuar precoce e preventivamente para evitar que os problemas na leitura dos alunos que os evidenciam se agravem.

b) Os alunos que nunca evidenciaram risco crescem mais semanalmente do que os alunos que evidenciaram risco em todo o ano letivo. Essa diferença vai ao encontro dos resultados obtidos por Deno et al. (2009) quando observou que os alunos em risco cresceram menos no decurso do ano escolar.

Os alunos que ficaram em risco todo o ano letivo crescem mais entre o período que vai desde o meio do ano até ao final do ano e nesse período exibem maior taxa de crescimento do que os alunos que nunca apareceram em risco. Já estes últimos crescem mais entre o período que vai da fase inicial do ano letivo até à fase intermédia. Segundo Vaz, Martins e Correia (2016) essa situação pode ter por base o facto de as dificuldades dos alunos que estão em risco impulsionarem um olhar mais atento por parte do professor e alguma alteração do tipo de estratégias utilizadas. Assim, constatando os 
problemas destes alunos na leitura, na fase inicial do ano letivo, possivelmente estará a promover um maior apoio e um maior investimento junto deles durante o restante ano escolar, não sendo, contudo, um apoio estruturado numa intervenção formal. Também os próprios alunos terão, eventualmente, maior consciência dos seus problemas o que pode desencadear naturalmente um maior esforço/empenho para os superar. No caso dos alunos que nunca evidenciam risco, e corroborando o referido por Christ et al. (2010), Deno et al. (2009) e Nese et al. (2012) eles evoluem na leitura principalmente até ao meio do ano, tornando-se esta evolução menor a partir daí pois, com efeito, tratando-se de alunos que nunca ficaram em risco já leem adequadamente logo, a partir desse momento o seu crescimento é menor.

c) Os problemas na articulação, o atraso no desenvolvimento da linguagem e a ausência de experiências de leitura a pares com os progenitores, destacam-se como fatores de risco na leitura. Esta situação corrobora Scarborough (1998) que associa os distúrbios específicos de linguagem a um risco elevado de dificuldades na leitura, e de Shaywitz (2008) que associa especificamente as dificuldades articulatórias ao aparecimento de graves dificuldades na leitura, por exemplo de Dislexia. Também Shapiro et al. (1990) e Shaywitz (2008) se referem às diferenças na aquisição e desenvolvimento da linguagem como fatores de risco na leitura.

Apesar de na maioria dos agregados familiares existir o hábito de leitura com as crianças, este traduz-se quase sempre na leitura da criança para o adulto. Na quase totalidade dos agregados familiares de alunos que se encontram em risco durante o ano letivo não existe a experiência de leitura a pares entre pelo menos um dos progenitores e o aluno, com o progenitor a ler para o seu educando e este de seguida para o progenitor, contando com o seu apoio quando necessário. Recorde-se a importância da leitura dos pais para a criança e desta para os pais no desenvolvimento da leitura nas crianças (PUT READING FIRST, 2000). Segundo Shaywitz (2008, p. 257). "para uma criança que se debate com a leitura, frequentemente é útil que lhe leia um pouco e, depois, a faça reler em voz alta o mesmo material cuja leitura acabou de modelar para ela".

Recorde-se que o sistema de ensino atual não contempla a identificação de fatores de risco em alunos que apresentam problemas na leitura e que se encontram em risco de verem os seus problemas agravarem-se. A sistematização que aqui acaba de se 
apresentar ajuda assim os professores a perceberem melhor os problemas que os alunos em risco apresentam e permite uma atuação preventiva sobre estes problemas que pode passar numa fase inicial pela "intervenção" ao nível de alguns dos preditores.

Contudo, a atuação perante os problemas na leitura que se esboça no contexto desta investigação não se esgota nos procedimentos aqui efetuados, ou seja, na implementação de um processo de triagem universal com a subsequente identificação de alunos em risco na leitura e respetiva caraterização no respeita aos fatores de risco neles presentes. É necessária a implementação de programas de intervenção ao nível da prevenção secundária e terciária tal como preconizados pelo Modelo de Resposta à Intervenção. 


\section{Referências}

BRADLEY, R.; DANIELSON, L.; HALLAHAN, D. P. Specific learning disabilities: Building consensus for identification and classification. In: BRADLEY, R.; DANIELSON, L., et al. (Ed.). Identification of learning disabilities: research to practice. New Jersey: Lawrence Erlbaum Associates, 2002.

BROWN, J.; SKOW, K. RTI: data-based decision making. [S.I.]: The Iris Center, 2009. BROWN-CHIDSEY, R.; DAVIS, L.; MAYA, C. Sources of variance in curriculum-based measures of silent reading. Psychology in the Schools, New Jersey, v. 40, n. 4, p. 363377, jul./set. 2003.

BURNS, S.; GRIFFIN, P.; SNOW, C. Starting out right: a guide to promoting children's reading success. Washington, DC: National Academic Press, 1999.

BUSCH, T. W.; LEMBKE, E. S. Teaching tutorial 5: Progress monitoring in reading using the CBM maze procedure. Division for Learning Disabilities of the Council for Exceptional Children, Califórnia, 2005.

CENTER RESPONSE TO INTERVENTION: at American Institutes for Research. Search: multi-level prevention system. Washington, DC: NCRI, 2012. Disponível em: <http://www.rti4success.org/categorycontents/multi-level_prevention_system>. Acesso em: 1 jan. 2012.

CHRIST, T. J. et al. Curriculum-based measurement of oral reading: An evaluation of growth rates and seasonal effects among students served in general and special education. School Psychology Review. [S.I.], v. 39, n. 3, p. 447-462, jul./set. 2010.

CLEMENS, N. H. et al. Tracing Student Responsiveness to Intervention With Early Literacy Skills Indicators: do they reflect growth toward text reading outcomes?. Reading Psychology. Filadélfia, v. 33, n. 1-2, p. 47-77, fev. 2012.

CORREIA, L. M. Inclusão e necessidades educativas especiais: um guia para educadores e professores. 2. ed. Porto: Porto Editora, 2013.

DAVIS, N.; LINDO, E.; COMPTON, D. Children at risk for reading failure: constructing an early screening measure. Teaching Exceptional Children. Califórnia, v. 39, n. 5, p. 32-37, maio. 2007.

DENO, S. L. et al. Basic academic skill samples: instructions for administration and a skill samples. Minnesota: United States Department of Education Grant, 1989.

DENO, S. L. et al. Developing a school-wide progress-monitoring system. Psychology in the Schools. Nova Jersey, v. 46, n. 1, p. 44-55, dez. 2009.

ESPIN, C. et al. Creating a progress-monitoring system in reading for middle-school students: tracking progress toward meeting high-stakes standards. Learning Disabilities Research \& Practice. [S.I.], v. 25, n. 2, p. 60-75, abr. 2010. 
FLETCHER, J. M.; VAUGHN, S. Response to intervention: preventing and remediating academic difficulties. Child Development Perspectives. Washington, v. 3, n. 1, p. 30-37, abr. 2009.

FUCHS, D. et al. Is "learning disabilities" just a fancy term for low achievement?: a metaanalysis of reading differences between low achievers with and without the label. In: BRADLEY, R.; DANIELSON, L., et al. (Ed.). Identification of learning disabilities: research to practice. New Jersey: Erlbaum, 2002a. p. 737-762.

FUCHS, L. S. Three conceptualisations of "treatment" in a responsiveness-to-treatment framework for LD identification. In: BRADLEY, R.; DANIELSON, L. et al. (Ed.).

Identification of learning disabilities: research to practice, New Jersey: Erlbaum, 2002b. p. 521-529.

FUCHS, L. S. Assessing intervention responsiveness: conceptual and technical issues. Learning Disabilities Research \& Practice. [S.I.], v. 18, p. 172-186, jun. 2003.

FUCHS, L. S.; FUCHS, D. The role of assessment within the RTI framework. In: FUCHS, D.; FUCHS, L. S., et al. (Ed.). Response to intervention: a framework for reading educators. Newark: international reading association. Newark, 2008. p. 27-49.

FUCHS, L. S.; STECKER, P. S. Progress Monitoring. Washington, DC, 2003. Disponível em: <http://www.studentprogress.org/weblibrary.asp-cbm_intro>. Acesso em: 16 set. 2009.

GARCÍA, I. M. Hiperactividade. Rio de Janeiro: McGraw-Hill, 2001.

GRANEY, S. B. et al. Universal screening of reading in late elementary school: R-CBM versus CBM maze. Remedial and Special Education. Califórnia, v. 31, n. 5, p. 368-377, set. 2010.

HUGHES, C.; DEXTER, D. D. Universal screening within a response-to-intervention model. [S.I.], 2013. Disponível em: <http://www.rtinetwork.org/learn/research/universalscreening-within-a-rti-model>. Acesso em: 18 dez. 2013.

JIMÉNEZ, R. R. et al. O deficiente auditivo na escola. In: BAUTISTA, R. (Ed.).

Necessidades Educativas Especiais. Lisboa: Dinalivro, 1997. P. 350-375.

JOHNSON, E. et al. Responsiveness to intervention (RTI): how to do it. National Research Center on Learning Disabilities. [S.I.], 2006.

MARTINS, A. P. L. Dificuldades de aprendizagem: compreender o fenómeno a partir de sete estudos de caso. 2006. 493 f. Tese (Doutoramento em Educação Especial)Universidade do Minho. Braga, 2006.

MCCARDLE, P.; SCARBOROUGH, H.; CATTS, H. Predicting, explaining, and preventing children's reading difficulties. Learning Disabilities Research \& Practice. [S.I.], v. 16, n. 4, p. 230, nov. 2001. 
MERCER, S. H. et al. Generalizability theory analysis of CBM maze reliability in thirdthrough fifth-grade students. Assessment for Effective Intervention. Califórnia, v. 37, n. 3, p. 183-190, jan. 2012.

NELP. National Institutute for Literacy. Developing early literacy: report of the national early literacy panel. Washington, DC., 2008.

NESE, J. F. T. et al. Within-year oral reading fluency with CBM: a comparison of models. Reading and Writing. África do Sul, v. 25, n. 4, p. 887-915, abr. 2012.

NJCLD. National Joint Committee on Learning Disabilities. Responsiveness to intervention and learning disabilities. [USA], 2005.

Learning disabilities and young children: identification and intervention.

Learning Disability Quarterly, Califórnia, v. 30, n. 1, p. 63-72, 2007.

PATRÃO, M. S. G. Monitorização com base no currciculo: um estudo quantitativo sobre a utilização de provas maze no contexto do nível I do modelo de atendimento à diversidade. 2010. 105 f. Dissertação (Mestrado em Educação Especial)-Universidade do Minho, Braga, 2010.

PIERCE, R.; MCMASTER, K.; DENO, S. L. The effects of using different procedures to score Maze measures. Learning Disabilities Research \& Practice. [S.I.], v. 25, n. 3, p. 151-160, ago. 2010.

PUT READING FIRST: Helping your child learn to read. Partnership for reading. [S.I.]: Jessup, MD, 2000.

RICHARDSON, R. D.; HAWKEN, L. S.; KIRCHER, J. Bias using Maze to predict high-stakes test performance among hispanic and spanish-speaking students. Assessment for Effective Intervention. Califórnia, v. 37, n. 3, p. 159-170, dez. 2012.

SCARBOROUGH, H. S. Early identification of children at risk for reading disabilities: phonological awareness and some other promising predictors. In: ACCARDO, P.; CAPUTE, A., et al. (Ed.). Specific reading disability: A view of the spectrum. [S.I.]: York Press, 1998. p. 75-119.

Developmental relationships between language and reading: reconciling a beautiful hypothesis with some ugly facts. In: CATTS, H. W.; KAMHI, A. G. (Ed.). The connections between language and reading disabilities. New Jersey: Erlbaum, 2005.

SCRUGGS, T. E. Alternatives to Rti in the assessment of learning disabilities. Kansas City, National Research Center on Learning Disabilities Responsiveness-to-intervention Symposium, 2003.

SHAPIRO, B. K. et al. Precursors of reading delay: neurodevelopmental milestones. Pediatrics. [S.I.], v. 85, n. 3, p. 416, mar. 1990.

SHAYWITZ, S. Vencer a dislexia: Como dar resposta às perturbações da leitura em qualquer fase da vida. Porto: Porto Editora, 2008. 
SHIN, J.; DENO, S. L.; ESPIN, C. Technical adequacy of the maze task for curriculum-based measurement of reading growth. Journal of Special Education. [S.I.], v. 34, n. 3, p. 164172, out. 2000.

SHINN, M. R.; SHINN, M. M. AIMSweb training workbook: administration and scoring of reading maze for use in general outcome measurement. [S.I.]: Eden Prairie, 2002.

SNOW, C.; BURNS, S.; GRIFFIN, P. Preventing reading difficulties in young children. Washington, DC: PRESS, N. A., 1998.

SPEECE, D. L. et al. Identifying children in middle childhood who are at risk for reading problems. School Psychology Review. [S.I.], v. 39, n. 2, p. 258-276, jun. 2010.

STANOVICH, K. E.; SIEGEL, L. S. Phenotypic performance profile of children with reading disabilities: A regression-based test of the phonological-core variable-difference model. Journal of Educational Psychology. Washington, DC, v. 86, n. 1, p. 24-53, mar. 1994.

STECKER, P. M.; SÁENZ, L.; LEMONS, C. Introduction to using CBM for progress monitoring in reading. Washington, DC: [s.n.], 2007.

THE IRIS CENTER: RTI: mathematics. [S.I.], 2010. Disponível em: <http://iris.Peabody.vanderbilt/module/rti-math>. Acesso em: 18 dez. 2013.

TICHÁ, R.; ESPIN, C.; WAYMAN, M. M. Reading progress monitoring for secondary-school students: reliability, validity, and sensitivity to growth of reading-aloud and mazeselection measures. Learning Disabilities Research \& Practice. [S.I.], v. 24, n. 3, p. 132142, ago. 2009.

VAUGHN, S.; BOS, C. Strategies for teaching students with learning and behavior problems. 7. ed. New Jersey: Pearson, 2009.

VAUGHN, S. R. Using response to treatment for identifying students with disabilities. In: BRADLEY, R.; DANIELSON, L., et al. (Ed.). Identification of learning disabilities: research to practice. New Jersey: Erlbaum, 2002. p. 549-554.

VAZ, P. M. F.; MARTINS, A. P.; CORREIA, L. M. Monitorização do progresso do aluno na identificação de risco na leitura. Cadernos de Pesquisa. São Paulo, v. 47, n. 164, p. 612630, abr./jun. 2016.

VAZ, P. M. F. Triagem universal de alunos em risco de apresentarem dificuldades de aprendizagem específicas na leitura: um estudo quantitativo no 3음 ano do 10 Ciclo do Ensino Básico. 2015. Tese (Doutorado)-Instituto de educação da Universidade do Minho, Braga, 2015. No prelo.

Recebido em: 07/02/2018

Aceito para publicação em: 25/06/2018 


\title{
Characterization of Students at Risk for Specific Learning Disabilities in Reading in a Universal Screening System
}

\begin{abstract}
This research aimed to characterize students at risk in reading, namely their level of understanding and intrinsic, parental and contextual risk factors associated with them. The research was carried out within a sample of 82 Portuguese students (third year). Data were collected with a curriculum-based measurement probe and a risk factors identification grid. The results allowed us to conclude that at the beginning of the academic year the mean results from students who were never at risk is three times higher than the mean results from those students who have been at risk throughout the year, and by the end of the academic year that difference is twice higher; the weekly growth rate of students who were never at risk is higher than the one from students who were at risk throughout the whole year; articulation problems, delay in language development and absence of reading experience in pairs with their parents stand out as reading risk factors.
\end{abstract}

Keywords: Universal screening. Reading. Risk factors. Curriculum-based measurement.

\section{Sistema de Selección Universal: Caracterización de Alumnos Identificados en Riesgo en Lectura}

\section{Resumen}

Esta investigación tiene por objeto caracterizar a los alumnos que se encuentran en riesgo en la lectura, en particular su nivel de comprensión y los factores de riesgo intrínsecos, parentales y contextuales asociados. Participaron 82 estudiantes portugueses de tercer grado. Los datos se recogieron a través de una prueba de monitoreo basada en el plan de estudios y en la rejilla de identificación de factores de riesgo. Se concluyó que, al comienzo del año escolar, los promedios de los resultados con respecto a la comprensión lectora de los estudiantes que nunca estuvieron en riesgo son superiores al triple de los que estaban en riesgo y al final del año escolar es aproximadamente el doble. La tasa de crecimiento semanal de los estudiantes que nunca estuvieron en riesgo es superior al de los estudiantes en riesgo durante el año escolar. Los factores de riesgo en la lectura más 
relevantes son los problemas en la articulación, el retraso en el desarrollo del lenguaje y la ausencia de lecturas en conjunto con los padres.

Palabras clave: Sistema de selección universal. Lectura. Factores de riesgo. Monitoreo basado en el plan de estudios. 